\title{
Generalized infection following facet joint injection -A case report-
}

\author{
Sae Young Kim, Sung Ho Han, Min Woo Jung, and Ji Hee Hong \\ Department of Anesthesiology and Pain Medicine, Keimyung University School of Medicine, Daegu, Korea
}

Facet joints have been shown to be a source of chronic low back pain, and it is generally accepted in clinical practice that diagnostic and therapeutic facet joint injections are the most reliable technique for the treatment of facet joint pain, which is considered to be an easy and safe procedure. Serious complications and side effects are uncommon after facet joint injection. However, infectious complications including septic arthritis, epidural abscess, meningitis and endocarditis have been reported following facet joint injections. We report here the first case of death following lumbar facet joint injection due to generalized infection. (Korean J Anesthesiol 2010; 58: 401-404)

Key Words: Abscess, Facet joint, Infection, Low back pain.

Many people suffer from low back pain, and its life-time prevalence rate has been reported to be up to $72 \%$ [1]. Among patients with chronic low back pain, $25 \%$ of cases are caused by a spinal facet joint [2]. Therefore, a facet joint injection (FJI) is a spinal interventional procedure commonly used for diagnostic purposes to determine if low back pain is caused by spinal facet joint and for therapeutic purposes to control the low back pain [3]. The procedure is used widely because of the relatively simple operational procedure without serious complications [4]. Carrino et al. [5] reported that more than 94,000 patients treated their low back pain using FJI and medial branch block (MBB) in 1999 in the United States. However, there are some reports on the development of infectious complications after receiving FJI. The authors encountered a case of death caused by multiple infections that developed from a septic embolism after performing FJI. We report this case with a review of the relevant literature.

\section{Case Report}

A 50 year old male patient suffered from a systemic febrile sensation, edema, and pain at his first day of admission. The patient had a prior history of disc replacement surgery using an artificial disc between his fifth lumbar vertebra and first sacral vertebra performed approximately 18 months earlier but no other specific findings were found. Ten days earlier, the patient visited hospital complaining of symptoms of aggravated low back and both buttock pain after lifting heavy loads. Therefore, the patient was diagnosed with spinal facet syndrome, and was treated with FJI, which alleviated the pain. The patient took NSAIDs and muscle relaxants to control the pain that had recurred 4 days before admission. However, the patient visited the emergency room due to the aggravated pain. The physical examination at admission revealed severe tenderness at his lumbar vertebral area, but no specific symptoms. The

Received: August 17, 2009. Revised: 1st, September 2, 2009; 2nd, September 7, 2009. Accepted: November $23,2009$.

Corresponding author: Sae Young Kim, M.D., Department of Anesthesiology and Pain Medicine, Keimyung University School of Medicine, Dongsan-dong, Jung-gu, Daegu 700-712, Korea. Tel: 82-53-250-7542, Fax: 82-53-250-7240, E-mail: mandell@naver.com

(c) This is an open-access article distributed under the terms of the Creative Commons Attribution Non-Commercial License (http:// creativecommons.org/licenses/by-nc/3.0/), which permits unrestricted non-commercial use, distribution, and reproduction in any medium, provided the original work is properly cited. 
hematologic test results was WBC $\left(11.4 \times 10^{9} / \mathrm{L}\right)$, ESR $(60 \mathrm{~mm} /$ hr), CRP (22.79 mg/dl), BUN (69.7 mg/dl), Creatinine $(2.5 \mathrm{mg} /$ dl), D-dimer $(1.01 \mu \mathrm{g} / \mathrm{ml})$, FDP $(5.0 \mu \mathrm{g} / \mathrm{ml})$, Fibrinogen $(717$ $\mathrm{mg} / \mathrm{dl}$ ), and Antithrombin III (52\%). The emergency lumbar spine MRI scanning test revealed an epidural abscess at the lower lumbar vertebral area with an abscess in both the left psoas major muscle and paraspinal muscle (Fig. 1). Compared to the MRI image taken 1 day before receiving the FJI, the newly developed abscess was observed by following the facet joints that had developed between the left third and fourth lumbar vertebra (L3-L4) and between the left fourth and fifth lumbar vertebra (L4-L5). Therefore, the patient was diagnosed with an epidural and paraspinal abscess, sepsis and acute renal failure. Along with the blood culture test, an intravenous injection of penicillin and cephalosporin antibiotics was started. Although percutaneous drainage was performed for the paraspinal abscess on the second day of hospitalization, it was not performed satisfactorily. Accordingly, epidural abscess debridement was performed under general anesthesia on the fourth day of hospitalization. A MRI of the edema and redness at the extremities revealed diffusible cellulitis at both of his lower extremities and at his right upper extremity along with an abscess at both feet and right elbow. Consequently, abscess drainage was performed. At the 10th day of hospitalization, the patient complained of pain around his sternal area, and the computed tomography (CT) revealed the development of a septic embolism and the formation of an abscess at the right second intercostal space, which was treated with abscess drainage by mediastinotomy. The surgical findings revealed extensive abscess formation that was linked to the mediastinum by following the right second intercostal space. Even after aggressive surgical abscess drainage and intravenous antibiotics injection treatment, the patient's condition did not improve and the sepsis was not interrupted. The patient died from multiple organ failure on the 32nd day of hospitalization.

The bacterial culture test of the abscess collected from patient's blood, urine, epidural space, extremities and mediastinum area revealed Staphylococcus aureus to be the causative organism.

\section{Discussion}

The spinal facet joint is an important factor that causes low back pain. Goldthwait first reported the facet joint to be an important cause of low back pain and instability of low back in 1911. In 1933, Ghormley defined the lumbosacral pain as "facet syndrome" regardless of the presence of sciatica that could developed after sudden rotary strain [6]. Generally, facet syndrome is not radiated lower than the knee, but tenderness can be present at the paraspinal muscle, which is characterized as pain that could be aggravated by the extension and rotation of the spine [7].

In 1963, Hirsch et al. reported recurrent pain when saline was injected into a spinal facet joint, which initiated the use of diagnostic FJI. Recently, the performance of FJI and MBB using local anesthesia can alleviate the pain in patients with chronic low back pain. Currently, the treatment of a facet joint is the second most commonly performed treatment for low back pain in the United States [6]. Although the procedure is frequently performed in such manner, coagulopathy, generalized infection, skin infection at the needle insertion points, hypersensitive reaction and an allergic response to drugs, pregnancy with a possible teratogenic effect by radiation, and the cases of percutaneously inaccessible spinal fusion operation that was performed for the target facet joint can be contraindications to the procedure [3]. Bleeding, infection, aggravating pain after the procedure, dural puncture with headache, drug allergy, and pneumothorax could be included as complications that can occur after performing FJI [3].
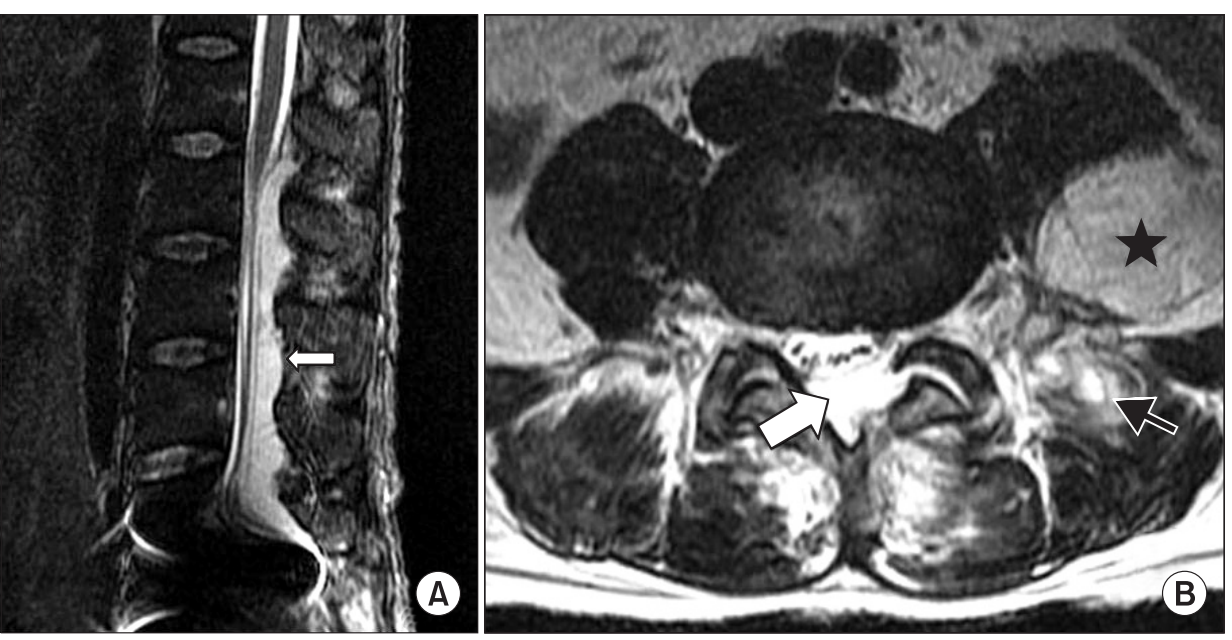

Fig. 1. Magnetic resonance imaging. T2-weighted sagittal images (A) and axial images (B) shows multifocal abscess formation of lower L-spine (white arrow), left lower back muscles (black arrow), left psoas muscle (star) and metal artifact at L5-S1 level. 
Among the infectious complications that can occur after FJI, there are 7 case reports of 8 patients [4,7-12]: 1 patient with an epidural abscess [7], 1 patient with a paraspinal abscess [8], 2 patients with septic arthritis accompanied with a paraspinal abscess [4,9], 2 patients showing the development of meningitis accompanied with paraspinal abscess [10], 1 patient with an epidural abscess accompanied with a paraspinal abscess [11], and 1 patient showing development of endocarditis accompanied with paraspinal abscess [12]. The patients who developed infectious complications had received FJI several times at more than one site. The current patient received FJI at 3 different sites. An epidural abscess has been reported to cause paralysis and sepsis in severe cases resulting in sudden death [13]. However, there are no reports of deaths occurring after FJI. In addition, the occurrence of such infectious complications is known to occur more prevalently in patients with diabetes mellitus or patients with immune-deficiency, which caused by alcohol and drug addiction [11]. Hence, clinicians should consider the potential for infection when performing FJI in such patients.

The following causative bacteria have been discovered during the infectious complications after performing FJI: Staphylococcus aureus in 5 patients, Staphylococcus epidermidis in 1 patient, and 2 cases in whom the causative bacteria could not be identified, which was reported to be caused by a pre-treatment using prophylactic antibiotics before performing the blood culture tests. Staphylococcus aureus was found to be the causative bacteria in the present case.

Muffoletto et al. [14] reported that Staphylococcus aureus is the most commonly detected bacteria in cases of naturally developed spinal facet joint infections that are spread by the blood stream, and they also suggested that percutaneous drainage ( $85 \%$ of success rate) and a single intravenous antibiotics injection ( $71 \%$ of success rate) as treatments. However, most cases of iatrogenic infections require surgical treatment. Orpen and Birch [9] recommended 6 to 8 weeks of intravenous antibiotics treatment for the spinal facet joint and paraspinal abscess followed by oral antibiotics treatment for 3 months.

Among the 8 patients who experienced an infection after receiving FJI, it is unclear if 2 patients had received treatment for the infection, but 4 patients received surgical treatment and intravenous antibiotics treatment simultaneously, 1 patient only received surgical abscess drainage without antibiotics treatment and 1 patient only received intravenous antibiotics treatment. Despite these aggressive treatments, only 1 of the 6 patients who had received treatment, experience sensory and motor dysfunction. In the current case report, the patient died as a result of sepsis despite the surgical and intravenous antibiotics treatments. All the patients who had experienced infectious complications after receiving FJI reported an aggravation of their low back pain or a change in their pain pattern. Among the
8 reported patients, 3 patients reported paraspinal tenderness, and 4 patients experienced symptoms of systemic infection including chills, fever and malaise.

The hematologic test results revealed an increase in the WBC count, ESR, and CRP levels, and the radiologic tests including MRI showed paraspinal and epidural abscesses with various inflammatory findings. Muffoletto et al. [14] reported that MRI provides high sensitivity and specificity only 2 days after the development of symptoms, and also added that the use of gadolinium contrast medium can be more helpful in making a diagnosis .

A failure of skin disinfection, an absence of sterile techniques, the use of contaminated catheters or needles, and cases of natural hematogenously disseminated infections could be included as possible causes of infectious complications after performing FJI. These infectious complications developed despite the use of complete aseptic procedures by practicing complete skin disinfection and using aseptic nerve block needles. As the cases that could be regarded as iatrogenic infections, the symptoms developed within a short time after FJI, 2 days to 20 days (mean 9.75 days) [10]. The time interval from the development of the first symptoms to the time point when treatment was started is the most important factor for the patient's prognosis, and immediate diagnosis and rapid treatment are essential [15].

To prevent the occurrence of infectious complications after FJI, diagnostic or therapeutic FJI need to be performed under a precise diagnosis made from the physical examinations and radiologic tests. It should be noted that performing FJI in too many sites or too frequently can increase the likelihood of complications. In addition, the procedure has to be performed aseptically by recognizing the fact that FJI could be a cause of fatal infectious complications. Since no infectious complications were reported after performing $\mathrm{MBB}$, it will be desirable to perform MBB for patients with a high probability of infection, and studies on the difference in infectious complications between the two procedures are warranted.

Hematologic tests, including WBC count, ERS, and CRP tests, should to be performed immediately when infection-suspicious symptoms are noticed, such as aggravated pain or a change in the pain pattern along with a febrile sensation and severe tenderness. MRI should be performed as soon as possible if an infection is suspected in the hematologic test results. After making a quick diagnosis, surgical or nonsurgical abscess drainage and immediate antibiotics treatment can reduce the occurrence of complications in these patients.

\section{References}

1. Jeffries LJ, Milaneses SF, Grimmer-Somers KA. Epidemiology 
of adolescent spinal pain: a systemic overview of the research literature. Spine 2007; 32: 2630-7.

2. Manchikanti L, Boswell MV, Singh V, Pampati V, Damron KS, Beyer CD. Prevalence of facet joint pain in chronic spinal pain of cervical, thoracic, and lumbar regions. BMC Musculoskelet Disord 2004; 5: 15.

3. Bogduk N. International spinal injection society guidelines for the performance of spinal injection procedures. Part 1: zygaphophyseal joint blocks. Clin J Pain 1997; 13: 285-302.

4. Weingarten TN, Hooten WM, Huntoon MA. Septic facet joint arthritis after a corticosteroid facet injection. Pain Med 2006; 7: 52-6.

5. Carrino JA, Morrison WB, Parker L, Schweitzer ME, Levin DC, Sunshine JH. Spinal injection procedures: volume, provider distribution, and reimbursement in the U.S. medicare population from 1993 to 1999. Radiology 2002; 225: 723-9.

6. Cohen SP, Raja SN. Pathogenesis, diagnosis, and treatment of lumbar zygapophysial (facet) joint pain. Anesthesiology 2007; 106: 591-614.

7. Alcock E, Regaard A, Browne J. Facet joint injection: a rare form cause of epidural abscess formation. Pain 2003; 103: 209-10.

8. Cook NJ, Hanrahan P, Song S. Paraspinal abscess following facet joint injection. Clin Rheumatol 1999; 18: 52-3.
9. Orpen NM, Birch NC. Delayed presentation of septic arthritis of a lumbar facet joint after diagnostic facet joint injection. J Spinal Disord Tech 2003; 16: 285-7.

10. Gaul C, Neundörfer B, Winterholler M. Iatrogenic (para-) spinal abscesses and meningitis following injection therapy for low back pain. Pain 2005; 116: 407-10.

11. Park MS, Moon SH, Hahn SB, Lee HM. Paraspinal abscess communicated with epidural abscess after extra-articular facet joint injection. Yonsei Med J 2007; 48: 711-4.

12. Hoelzer BC, Weingarten TN, Hooten WM, Wright RS, Wilson WR, Wilson PR. Paraspinal abscess complicated by endocarditis following a facet joint injection. Eur J Pain 2008; 12: 261-5.

13. Rombauts PA, Linden PM, Buyse AJ, Snoecx MP, Lysens RJ, Gryspeerdt SS. Septic arthritis of a lumbar facet joint caused by Staphylococcus aureus. Spine 2000; 25: 1736-8.

14. Muffoletto AJ, Ketonen LM, Mader JT, Crow WN, Hadjipavlou AG. Hematogenous pyogenic facet joint infection. Spine 2001; 26: 1570-6.

15. Kindler CH, Seeberger MD, Stanender SE. Epidural abscess complicating epidural anesthesia and analgesia. An analysis of the literature. Acta Anaesthesiol Scand 1998; 42: 614-20. 